\title{
Inhalt - Contents, Vol. 11, No. 3, 1995
}

\section{Editorial}

Schönleben, K.

Chiruigische Gastroenterologie

Contents

Editorial

,201 Schönleben, K.

\section{$\left.\mathrm{K}^{3} / 4\right\rangle \omega \ll$}

,201

Melullis, M., Birth, M., Weiser, H.-F. 203 Melullis, M., Birth, M, Weiser, H.-F.

Teil I: Hauptthema

Laparoskopische Cholezystektomie: Aktuelle Empfehlungen zur prä- und intraoperativen

Diagnostik und unmittelbare operative Konsequenzen

Part I: Main Theme

Laparoscopic Cholecystectomy: Topical Recommendations on the Pre- and Intraoperative Diagnostics and Its Operative Conclusions

,203

Cholezysto- Choledocholithiasis: Laparoskopische Gesamtlòsung oder therapeutisches Splitting? Zender, F. J., Schönleben, K 210

Cholecysto-Choledocholithiasis: Complete Laparoscopic Solution or Therapeutical Splitting?

Zender, F. J., Schönleben, K 210

Langzeitergebnisse nach laparoskopischer Cholezystektomie: Halt die Operation, was sie verspricht?

Eypasch, E., Ure, B., Lefering, R., Troidl, H. ...

,218

Long-Term Results after Laparoscopic Cholecystectomy: Does the Operation Fulfil Its Promise?

Eypasch, E., Ure, B., Lefering, R., Troidl, H.

.218

Laparoskopische Hiatushernien-/Antirefluxchirurgie: Methodische Empfehlungen - derzeit bewährte Technik

Fuchs, K.-H., Heimbucher, J., Freys, S. M., Fein, M 224

Laparoscopic Hiatal Hernias-/Antireflux Surgery: Methodical Recommendations - Presently Established Technique

Fuchs, K.-H., Heimbucher, J., Freys, S. M., Fein, M 224 
Laparoskopische Operationen beim Gastroduodenal-ulkus - elektiver Eingriff: Kritische Bewertung des methodischen Angebots und Analyse der ersten Ergebnisse

Bodner, E., Sattler, J., Keiler, A 232

Laparoscopic Operations for Duodenal Ulcer Disease - Elective Surgical Intervention: Critical Evaluation of Methods and Analysis of First Results

Bodner, E., Sattler, J., Keiler, A

.232

Wertigkeit der laparoskopischen Therapie des perforierten peptischen Gastroduodenalulkus Schönleben, K., Zittel, F.-U., Rheinwalt, K.-P.

Evaluation of Laparoscopic Surgery in the Treatment of Perforated Gastroduodenal Ulcers ,241 Schönleben, K, Zittel, F.-U., Rheinwalt, K.-P.

.241

Laparoskopische Anastomosierungsverfahren am Dickdarm: Kritische Bewertung aus Tierexperiment und klinischer Praxis

Köckerling, F, Schneider, C, Reck, Th 246

Laparoscopic Large-Bowel Anastomoses:

Critical Assessment Based on Animal Experiments

and Clinical Practice

Köckerling, F, Schneider, C, Reck, Th

,246

Laparoskopische Dickdarmresektion: Indikationen -Patientenselektion - bisherige Ergebnisse Hildebrandt, U., Feifel, G 252

Laparoscopic Colon Resection: Indications -Patient Selection - Present Results

Hildebrandt, U., Feifel, G

252

Band 11, Heft 3, September 1995

Chirurgische Gastroenterologie

Inhalt

Contents

Argumente pro laparoskopische Appendektomie

Thiele, H., Lang, R. D

Arguments for Laparoscopic Appendectomy

. 258 Thiele, H., Lang, R. D

.258

Argumente kontra laparoskopische Appendektomie Arguments against Laparoscopic

Appendectomy

Rückert, K., Niebuhr, H., Nahrstedt, U 262 Rückert, K., Niebuhr, H., Nahrstedt, U 262

Diagnostische und therapeutische Sicherheit bei der Appendizitis - Kommentar zu den

vorstehenden Arbeiten von H. Thiele und R. D. Lang sowie K. Rückert et al.

Feifel, G

Wertigkeit laparoskopischer Untersuchungs-techniken bei malignen Erkrankungen 
Feussner, H., Kraemer, S. M., Siewert, J. R

Diagnostic and Therapeutic Reliability in Case of Appendicitis: Commentary to the Preceding Articles by H. Thiele and R. D. Lang, and K. Rückert et al.

266 Feifel, G

Importance of Laparoscopic Procedures in Malignant Diseases

. 268 Feussner, H., Kraemer, S. M., Siewert, J. R. .

,266

.268

Laparoskopische Leistenhernienreparation: Transperitoneal- Laparoscopic Hernia Repair:

Transperitoneal or

oder extraperitoneal? Erkenntnisstand heute Extraperitoneal? Level of Knowledge Today

Kunz, R., Link, K. H 274 Kunz, R., Link, K. H

.274

Teil II: Interdisziplinäres Gespräch

Minimal invasive Chirurgie: Heutiger Standard und Grenzen

Büchler, M.W, Säuberli, H. (Gesprächsleiter)

278

Part II: Interdisciplinary Discussion

Minimally Invasive Surgery: Today's Standard and Limits

Büchler, M.W, Säuberli, H. (Discussion Leaders)

278

Impressum

Buchbesprechungen .. Hinweise für Autoren

. 198 Imprint 198

.286 Book Reviews 286

.291 Instructions to Authors 291 